\title{
Fast In Situ Nanotomography at ESRF
}

Julie Villanova $^{1}$, Richi Kumar ${ }^{1,2}$, Rémi Daudin ${ }^{2}$, Pierre Lhuissier ${ }^{2}$, David Jauffrès ${ }^{2}$, Christophe L. Martin $^{2}$, Rémi Tucoulou ${ }^{1}$, Sylvain Labouré ${ }^{1}$, Gema Martinez-Criado ${ }^{1-3}$, Luc Salvo ${ }^{2}$

${ }^{1}$ ESRF The European Synchrotron, CS 40220, 38043 Grenoble Cedex 9, France

${ }^{2}$ Univ. Grenoble Alpes, CNRS, SIMaP, F-38000 Grenoble, France

${ }^{3}$ Instituto de Ciencia de Materiales de Madrid, Consejo Superior de Investigaciones Científicas, 28049Cantoblanco, Spain

*Corresponding author: julie.villanova@esrf.fr

X-ray computed tomography (CT) is a very powerful technique that provides nondestructively direct access to the three-dimensional morphology of a specimen. It is extensively used in different domains like medicine, paleontology and biology. Today it is even possible to perform real time acquisition to observe in situ damaging and processes occurring in materials. Thanks to continuous improvements in spatial resolution, CT has been recently extended to the nanoscale [1]. It is a very efficient technique to study the performance of many advanced materials, which is mostly determined by the arrangement of their nanostructure [2]. Nevertheless, the combination of fast real time, in situ and CT in the nanometer regime remains a key challenge. We present here, in situ hard X-ray nanotomography experiments with an unprecedented combination of nanometer pixel size and fast acquisition time at high temperature.

ID16B is a nano-analysis beamline built during phase I of the European Synchrotron Radiation Facility (ESRF) and opened to users since early 2014. The beamline configuration offers an improved lateral resolution $\left(50 \times 50 \mathrm{~nm}^{2}\right)$, a monochromatic nanobeam tunable in a large energy range $(6-30 \mathrm{kV})$ with high flux $\left(10^{9}\right.$ to $\left.10^{12} \mathrm{ph} / \mathrm{s}\right)$, and large flexibility capable of in situ experiments [3]. Combination of various techniques such as X-ray fluorescence (XRF), X-ray absorption (XANES), X-ray excited optical luminescence (XEOL) and X-ray diffraction (XRD) as well as magnified phase contrast nanotomography allows multi-approach studies to be performed. Taking advantage of the high stability offered on ID16B and magnified phase contrast imaging, we developed a fast in situ nanoimaging set-up [4] that allows high temperature experiments to be performed with an unparalleled combination of nanometer pixel size $(35 \mathrm{~nm})$ and fast acquisition $(<10 \mathrm{~s})$. An intense X-ray beam $\left(10^{12} \mathrm{ph} / \mathrm{s}\right)$ is focused down to a spot size of $50 \times 50 \mathrm{~nm}^{2}$ using two multilayer-coated Si mirrors in Kirkpatrick-Baez (KB) configuration under pink beam mode $\left(\Delta \mathrm{E} / \mathrm{E}=10^{-2}\right)$. As illustrated in Figure 1 the sample located in the microheater $\left(200\right.$ to $\left.900^{\circ} \mathrm{C}\right)$ is positioned out of the focal plane in projection geometry. While rotating the sample, high-resolution images are continuously collected by a PCO edge 5.5 detector (equipped with a CMOS sensor) through an Optique Peter high resolution X-Ray imaging microscope (equipped with an X10 Olympus objective). To date, fastest full tomographic scans are acquired in 7s. Pixel size and field of view depend on the sample position between the focal plane and the detector. Any pixel size between 27 and $650 \mathrm{~nm}$ is available and multi-scale measurements are easily achievable.

Several real time studies that investigates nanostructure evolution in materials, have been already performed: (i) ceramics sintering (ii) light alloys damage (iii) fuel cells degradation... For example, spherical soda-limes glass particles have been isothermally treated at $670^{\circ} \mathrm{C}$, and continuously scanned (timescan:33s) during approximately 2 hours with a pixel size of $100 \mathrm{~nm}$. The microstructural evolution during ceramic powders sintering leads to controlled porosity but also formation of defects. 3D observations of the process at the micro-scale already gave important information on the densification process from rearrangement phenomena point of view. While the $3 \mathrm{D}$ movie resulting from in situ 
nanotomography allows early stages of the sintering to be observed. It has revealed two necks population. The two neck groups exhibit different growth rates and both do not fit the classical Frenkel model, which predicts a neck size that scales with the square root of time. Moreover, thanks to the high resolution, neck curvature evolution, which is an essential parameter for sintering process, has been analyzed and invalid the basic tangent-circle approximation classically used to build densification models.

Another example relates to remelting phenomena that occurs during new additive manufacturing for light alloys forming. Indeed, at the semi-solid state, liquid droplets are formed and become intermetallics once the alloy is cooled down. If the presence of liquid droplets is commonly observed, the mechanism of these droplets formation, such as the nucleation sites and coalescence remains unclear. An aluminium with $2.6 \mathrm{wt} \%$ of copper has first been solutionized for two hours at $540^{\circ} \mathrm{C}$ and then reheated at $3{ }^{\circ} \mathrm{C} / \mathrm{min}$ in the semisolid range up to $625^{\circ} \mathrm{C}$. The last step of the process has been carefully observed by acquiring 20s scans with a pixel size of $100 \mathrm{~nm}$ during $20 \mathrm{~min}$. 3D reconstructed images enable a full tracking of the liquid droplets formation during the partial remelting of the alloy: from the nucleation up to growth and coalescence. Size distribution, number vs time as well as "critical" nucleation size have been determined, leading to a first answer to the yet unsolved question: is the nucleation homogenous or heterogeneous?

The fast in situ nanotomography developed at ID16B that allows real time measurements of thermal driven phenomena fills the gap in the pixel size vs acquisition time for in situ tomography. It has been already used for several high temperature studies in materials science but thanks to continuous improvements, the technique can be also used today to study operando process as for example battery cycles and fuel cells degradation. In the future, the capabilities of the current experimental setup will be further increased thanks to the EBS upgrade program at the ESRF as well as the development of other devices such as loading systems and furnaces reaching higher temperatures.

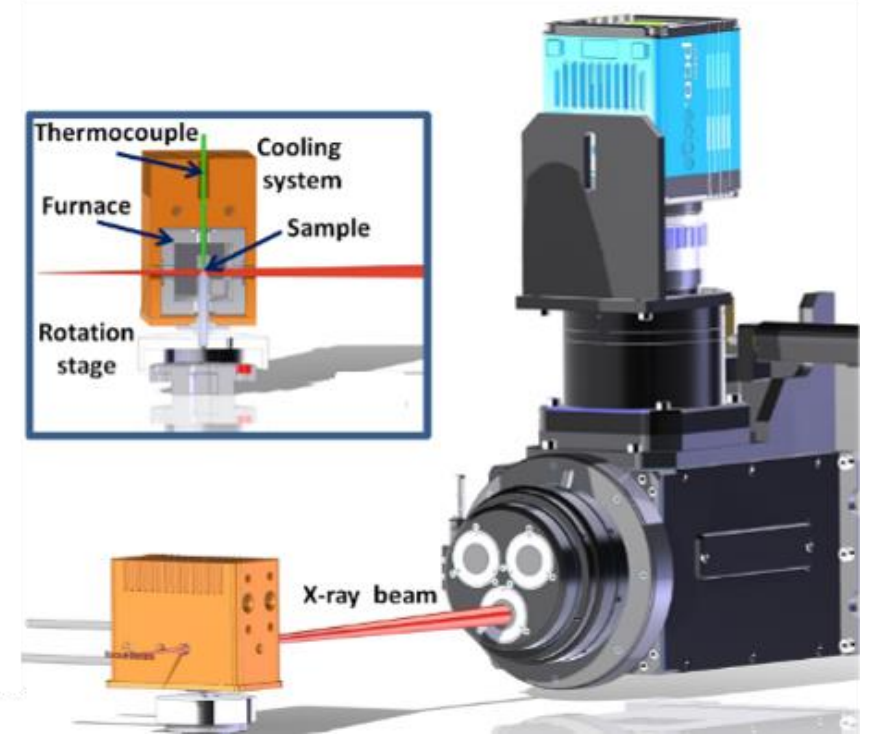

Figure 1. Schematic representation of the high temperature fast in situ nanotomography set up developed at ID16B ESRF beamline. The X-ray imaging microscope allows three scintillators to be used depending on acquisition conditions. The inset shows a cross section of the micro-heater exposing the cooling system, resistive furnace, quartz chamber and thermocouple.

References:

[1] D. Attwood, Nature 442 (2006) 642.

[2] E. Maire, P.J. Withers, Int. Mater. Rev. 59(1) (2014) 1.

[3] G. Martínez-Criado et al, J. Synchrotron Radiat. 23 (2016) 344.

[4] J. Villanova et al, Mater. Today 20 (2017) 354. 